\title{
THE EUROPEAN REGISTER OF CERTIFIED PHARMACOLOGISTS AS AN INITIATIVE TO SUPPORT THE PROFESSION OF PHARMACOLOGIST: THE ITALIAN EXPERIENCE
}

\author{
D. Grisafi ${ }^{1,6}$, A. Capuano ${ }^{2,6}$, M. Sortino ${ }^{3,6}$, M. Grilli ${ }^{4,6}$, F. Drago $3,5,6$ \\ ${ }^{1}$ Order of Pharmacists of Padua's Province, Italy \\ ${ }^{2}$ Centro Regionale di Farmacovigilanza Regione Campania, L. Vanvitelli University of Campania, Napoli, Italy \\ ${ }^{3}$ Department of Biomedical and Biotechnological Sciences, School of Medicine, Catania, Italy \\ ${ }^{4}$ Laboratory of Neuroplasticity Department of Pharmaceutical Sciences, University of Piemonte Orientale, \\ Novara, Italy \\ ${ }^{5}$ Clinical Pharmacology Unit, University Hospital, Catania, Italy \\ ${ }^{6}$ Italian Society of Pharmacology (SIF), Milan, Italy
}

E-mail: f.drago@unict.it

Doi: $10.36118 /$ pharmadvances.2021.14

\begin{abstract}
SUMMARY
As of today, pharmacologists should have experience in the various stages of drug development in basic and clinical research, from the laboratory bench to the patient's bedside. The recent WHO position paper on the role of Pharmacology in healthcare, teaching and research has clarified its positive impact on healthcare systems. Over the last 40 years, Pharmacology has advanced in European countries, where extensive activity has led to the constitution of the European Association of Clinical Pharmacology and Therapeutics (EACPT) and the Federation of European Pharmacological Societies (EPHAR). A program envisioned by EPHAR, The European Certified Pharmacologist (EUCP) initiative was developed jointly with EACPT in 2014, with the specific aim of identifying individuals working in the field of Pharmacology who excel in terms of educational standards, skills, experience and professional standing. European Certified Pharmacologists must have demonstrated that their competence profile, in addition to their personal specialized scientific expertise, encompasses expertise in all major fields of Pharmacology and that they have experience and practical knowledge in a broad spectrum of pharmacological techniques. To promote EUCP in Italy, the Italian Society of Pharmacology (SIF) has established in 2018 a committee that worked on the definition of the Italian criteria for the admission of candidates to the program named "SIF-EuCP". This initiative should stimulate a stronger position of Italian and European Pharmacology towards political entities, professional organizations, foundations and scientific stakeholders in gaining public support for pharmacology and awareness of the importance of this science in providing advice and expertise.
\end{abstract}

\section{Key words}

Pharmacology; certification; health professionals; SIF-EUCP.

\section{Impact statement}

The SIF-EuCP initiative supports the European certification of health professional or academic pharmacologists to provide an official inventory that is accessible to public and private health institutions. 
Pharmacology is a multidisciplinary discipline encompassing clinical and non-clinical aspects of the relationship between drugs and human beings and involving professionals with a wide variety of scientific expertise including medicine, pharmacy, and biomedical sciences (1). The term "pharmacologist" is commonly used in a professional sense to refer to physicians, biomedical scientists and/or pharmacists of $\mathrm{PhD}$ level, with proven experience in improving patient care, directly or indirectly, by developing better or novel drugs and promoting a safer and more effective use of medicines (2). Therefore, pharmacologists teach, undertake research activities, formulate policies, provide information and advice on the correct mechanism of action, and use of medicines in humans and, together with clinicians, implement this knowledge in the clinical practice.

As of today, a pharmacologist is required to have experience not only in various stages of drug discovery and development in basic and clinical research, but also in regulatory affairs for institutions, clinical research organizations, government agencies and pharmaceutical industries, including the biotechnology sector (3).

Several specific skills are required for a clinical pharmacologist. Among them, good knowledge of molecular pharmacology, experience and in-depth knowledge of clinical development methodologies and pharmacokinetics, strong understanding of quality requirements (GCP/ICH) in clinical drug development, understanding of bio-statistical analysis concepts, ability to analyze, interpret, present, and publish results of clinical studies. In addition, strong academic (international) background, good networking with academic and professional societies and industrial organizations, teamwork ability to work effectively with a cross section of people, represent mandatory skills (4).

To date, pharmacologists face several challenges in their basic science and clinical applications: advanced therapy medicinal products and biologics certainly represent some of them, but not the only ones. There is also a problem of communicating and informing people about scientific advances, and pharmacologists can help to develop scenarios and offer the right answers to demographic issues such as growing and ageing populations, historical and emerging infectious diseases, and the fight against cancer (5). The WHO position paper on their key roles in health care, teaching and research has clarified the positive impact that a clinical pharmacologist provides in the hospital setting (6).

In the last decades, Pharmacology has made advances in the EU where an extensive discussion took place resulting in the institution of the European Association of Clinical Pharmacology and Therapeutics (EACPT) and the Federation of European Pharmacological Societies (EPHAR).

The EACPT initiative, launched in 1993 at a meeting in Verona, Italy, includes EU members National organizations for Clinical Pharmacology and provides educational and scientific support for professionals interested in clinical pharmacology and therapeutics throughout the European region (7).

EPHAR was founded on occasion of the XI International Congress of Pharmacology in Amsterdam, The Netherlands, in 1990. Since its establishment, EPHAR aim was to advance research and education in Pharmacology and related sciences and to promote cooperation between National/Regional Pharmacological Societies in Europe. In 1994, EPHAR was recognized as an affiliate member of International Union of Basic and Clinical Pharmacology (IUPHAR) (8).

EPHAR is focused on congressional activities and networking among pharmacologists. These will include plenary lectures, symposia, round tables, oral and poster communications devoted to the most recent advances in Pharmacology and related sciences, therefore representing the adequate forum for discussing preclinical, clinical, and therapeutic data. There is also a relevant attention to politics of Pharmacology and specifically the relation- 
ship between science and society in Europe, with regards to drug registration, strategies for research funding and training of pharmacologists (History of EPHAR, 1993).

An interesting initiative developed by EPHAR, in conjunction with EACPT, in 2014 is The European Certified Pharmacologist (EuCP). Specific aim of the EuCP program is identifying individuals working in the field of Pharmacology who excel in terms of educational standards, skills, experience, and professional standing. EuCP need to have demonstrated that their competence profile, in addition to their personal specialized scientific experience, cover expert knowledge in all major fields and that they have experience and practical awareness in a broad spectrum of pharmacological techniques. The system therefore ensures that common high standards for this certification are applied by all participating Societies of Pharmacology across Europe https://www.eucp-certification.org/documents.html (9).

The EuCP, similarly to the certification platform for the European Register of Toxicologists, EUROTOX, instituted in 1994 (ERT_GUIDELINES Main_text_2016), requires applicants to follow guidelines, approved by each national Pharmacological Society, that describe the formal requirements and procedures for certification and recertification, as well as the fields of theoretical and practical knowledge and experience that are relevant for eligibility for such certification.

The EuCP program also provides incentives to encourage individual scientists to expand their personal skills profiles so as to increase their chances of obtaining high-level positions in an increasingly competitive working environment, whether academic, industrial, regulatory, or self-employed. The need for continuous professional development and expansion of personal competencies was already identified at pan-European level by the European Union's Innovative Medicines Initiative (IMI), which, with a total financial volume of more than $€$ 5.3 billion, is the largest European public-private partnership jointly funded by the EU and the European Federation of Pharmaceutical Industries and Associations (EFPIA) (https:// www.imi.europa.eu/).

The EuCP certification procedure is essentially a two-step process. The national certifying body (a National Pharmacology or Clinical Pharmacology Society) handles applications from individuals, assesses the applications according to a consensus process, based on the criteria and procedures described in the certification guidelines, and verifies the credentials of the candidates. Successful candidates are then nominated by the EuCP Committee which will issue the certification as European Certified Pharmacologist. To ensure continuous professional development (CPD), certification must be renewed at regular intervals upon submission to the certifying body of evidence of personal CPD activities. Currently there are EuCP programs approved in five European countries: Italy, Austria, the Netherlands, Switzerland, and Portugal.

In 2018, the Italian Society of Pharmacology (SIF) established a committee that discussed and identified the Italian criteria for EUCP candidate admission. In 2020, the certification system, named "SIF-EuCP", has become operative to meet the requests of all those who work in the field of Pharmacology in Italy, and of young pharmacologists who are open to European horizons as a new scenario of professional opportunities.

The program consists of two pathways: one dedicated to the candidate with a professional career in academia and the other one dedicated to the health professional working in the pharmaceutical industry or in public/private healthcare systems (public hospitals, private nursing homes, hospital pharmacies, contract research organizations). The SIF-EuCP certification scheme is detailed in figure 1.

Specific checklists (Annexes I and II) are used by the certifying committee to evaluate the candidate's competences by assigning a specific score for each capacity (figure 2).

Although the program has just started, several promotional activities have been planned 


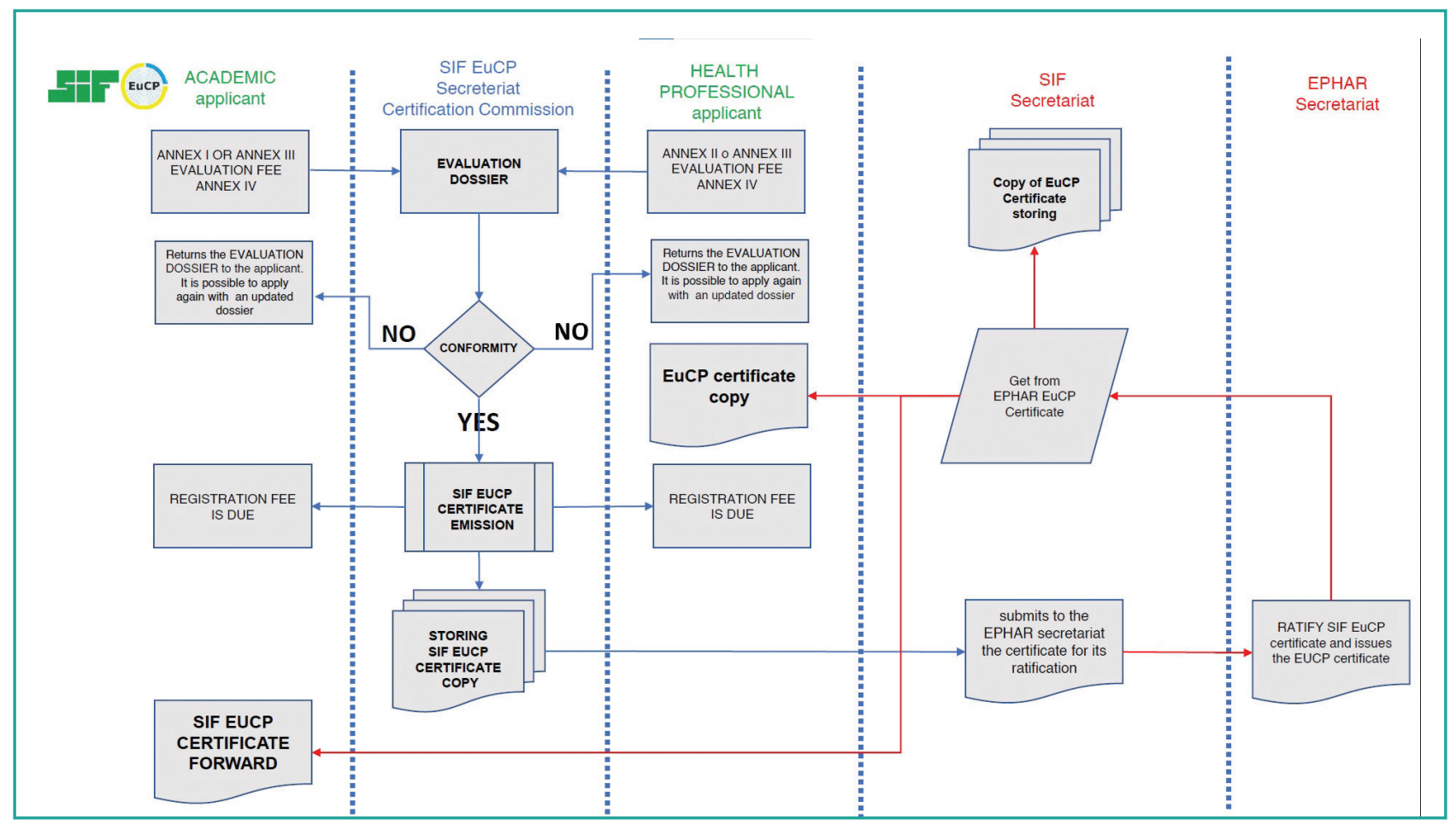

Figure 1. SIF-EuCP certification scheme pathways: one dedicated to the candidate with a professional career in academia and the other one dedicated to the health professional working in the pharmaceutical industry or in public/private healthcare systems.

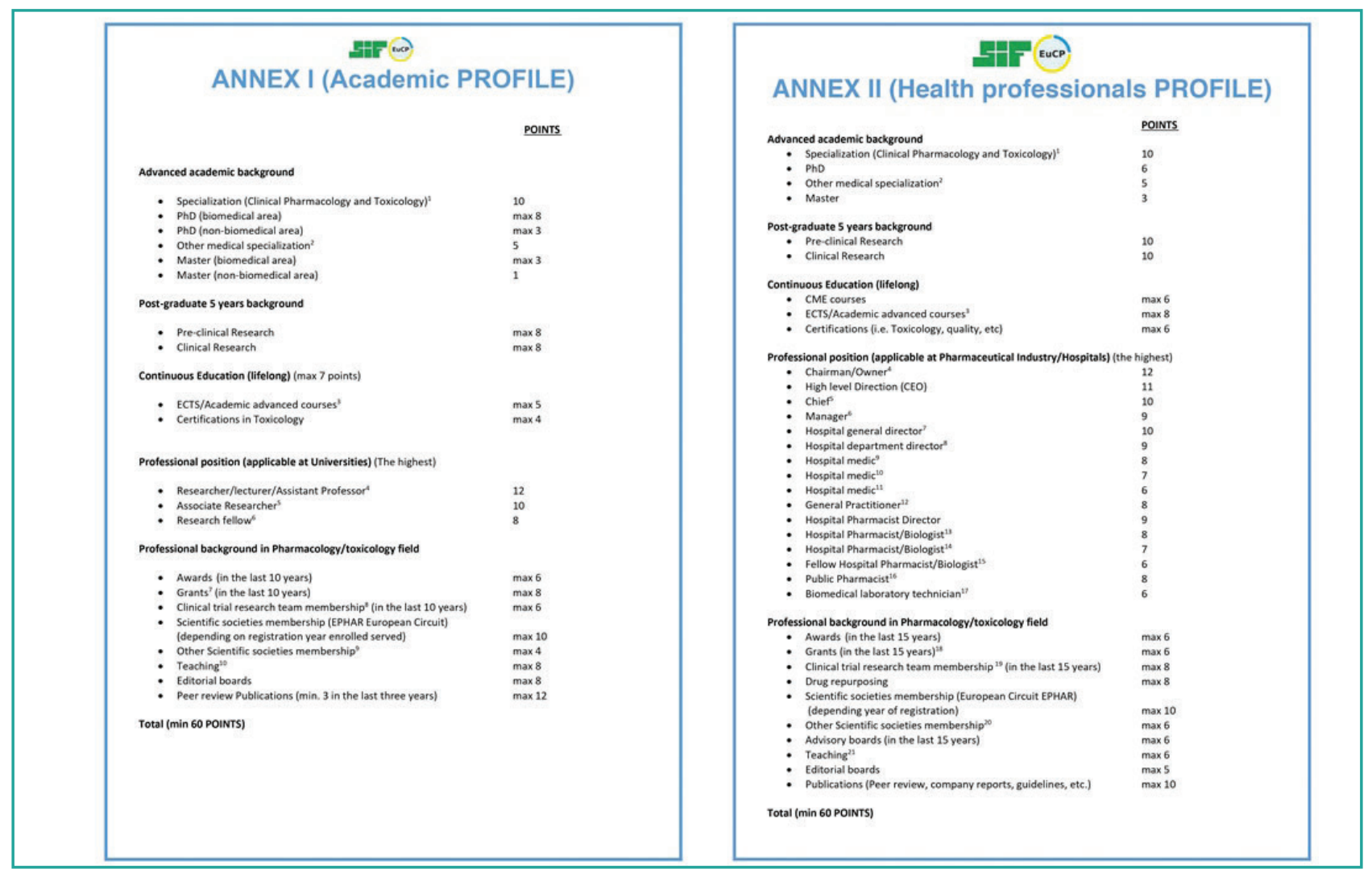

Figure 2. Annexes I and II are specific checklists used by the certifying committee to evaluate the candidate's competences by assigning a specific score for each capacity. 
and are ongoing, involving institutional, academic, and pharmaceutical industries. Recently, on occasion of the $40^{\text {th }}$ Congress of the Italian Society of Pharmacology (March 9-13, 2021), a symposium was fully dedicated to the Italian experience on EuCP certification (10).

The SIF-EuCP program is expected to reinforce the sense of identity of pharmacologists as it represents a new resource for those working in the field of Pharmacology as an integration of professional expertise within an evolving $\mathrm{Na}$ tional Health System. The SIF-EuCP program could also promote and add value to the key role of clinical pharmacologists in the context of the National Healthcare System since in some European countries (including Italy) this is not yet established.

Indeed, in many European universities, departments of Pharmacology have been abolished or merged with other units or, even worse, the teaching of Pharmacology has been abolished to assign some of its rudiments to lecturers in related areas. Many pharmacologists have therefore rightly felt the strong need to identify themselves as such through membership of their National Society of Pharmacology and international certification. Therefore, the SIF-Eu-

\section{REFERENCES}

1. Gaddum JH. Clinical Pharmacology. Proc R Soc Med 1954;47(3):195-204.

2. Birkett D, Brøsen K, Cascorbi I, et al. Clinical pharmacology in research, teaching and health care: Considerations by IUPHAR, the International Union of Basic and Clinical Pharmacology Basic Clin Pharmacol Toxicol 2010;107(1):531-59.

3. Martin JH, Henry D, Gray J, et al. Achieving the World Health Organization's vision for clinical pharmacology. $\mathrm{Br} \mathrm{J}$ Clin Pharmacol 2016;81(2):223-7.

4. Richards D. Developing and delivering clinical pharmacology in pharmaceutical companies. Br J Clin Pharmacol 2012;73(6):870-3.
CP program could increase the visibility of our science and, consequently, a new and more effective positioning of the pharmacologist in the various contexts of the European scientific and economic world.

This is likely to result in a much stronger position of Italian and European Pharmacology vis-à-vis political entities, professional organizations, foundations, and scientific stakeholders when it comes to obtaining public support for pharmacological research and awareness of the importance of our science in providing advice and expertise.

\section{ACKNOWLEDGEMENTS}

The authors would like to thank the Italian Society of Pharmacology (SIF) for sponsoring the publication of this paper.

D.G. and F.D. conceived of the presented idea. M.G., M.S. and A.C. supervised the findings of this work. All authors discussed the results and contributed to the final manuscript.

\section{CONFLICT OF INTERESTS}

The authors declare that they have no conflict of interests.

5. Grisafi D, Ceschi A, Sava G, Avalos Clerici V, Scaglione F. Reconsidering clinical pharmacology frameworks as a necessary strategy for improving the health care of patients: a systematic review. Eur J Clin Pharmacol 2018;74(12):1663-70.

6. World Health Organization. Clinical Pharmacology in Health Care, Teaching and Research. Position Paper 2012. Accessed April 30, 2021.

7. Orme M European Association of Clinical Pharmacology and Therapeutics. Br J Clin Pharm 1993;36:183-4.

8. History of Ephar. Available from https:// www.ephar.org/federation.html. Accessed April 30, 2021. 
9. The European Certified Pharmacologist (EuCP). Available from https://www.eucp-certification.org/. Accessed April 30, 2021.

10. Ceschi A, Drago F, Griesbacher T, Kraneweld AD. The certification in Pharmacol- ogy: the Italian way. (Online Webinar Scientific session, Mar 11, 2021). $40^{\circ}$ Congresso Nazionale della Società Italiana di Farmacologia. 